\title{
Characterization of the chromatin of some liliaceous species after digestion with restriction endonucleases and sequential Giemsa, fluorochrome and silver staining
}

\author{
R. Lozano, \\ M. Jamilena, \\ C. Ruiz Rejón and \\ M. Ruiz Rejón
}

\begin{abstract}
Departamento de Biología Animal, Ecología y Genética, Facultad de Ciencias, Universidad de Granada, 18071 Granada, Spain.
\end{abstract}

\begin{abstract}
A good C-banding pattern or a modified C-banding pattern is obtained after digestion with some restriction endonucleases (REs) on the fixed metaphase chromosomes of four liliaceous species. The C bands of $A$ llium subvillosum and Muscari comosum are resistant to digestion with some $R E$ shose recognition sequences are either GC- or AT-rich. The telomeric C-bands of $A$. cepa are resistant not only to some endonucleases whose recognition sequences are absent in the DNA satellite existing in these regions, but also to some endonucleases for which there exist numerous recognition sequences. However, the GC-rich C-band associated to the NOR of Scilla autumnalis is entirely digested by Hae III, and some of the faint GC-rich C-bands of the three NORs of A. subvillosum are digested by Hae III, Alu I or Rsa I. The action of endonucleases on plant chromosomes in general, and particularly on heterochromatin and NORs, together with the usefulness of these enzymes in cytogenetic analyses of plants, are discussed.
\end{abstract}

\section{INTRODUCTION}

The development of restriction endonuclease banding over recent years has yielded a valuable tool for chromosome analysis to add to the numerous banding techniques which were previously available. This new banding method has already been applied to the chromosomes of an extensive variety of animal species (see for instance Schmid and Almeida, 1988). However, the only plant species cytologically analysed by means of restriction endonucleases, at least to our knowledge, is Vicia faba (Frediani et al., 1987). After digestion of fixed animal chromosomes with REs two conclusions seem clear: (1) the enzymes selectively digest chromosomal DNA, and (2) the enzymes act on chromosomes regardless of the organizational compactness of specific areas. In relation to the first point, $\mathrm{G}$ - and $\mathrm{C}$-banding (or modified G- and C-banding) are demonstrated in warm-blooded vertebrates after digestion with restriction enzymes, while only C-bands are shown in cold-blooded vertebrates and insects. In relation to the second point, restriction enzymes have been found to digest not only the euchromatic regions but also certain regions of constitutive heterochromatin of many animal chromosomes. For instance, the mouse $\mathrm{Y}$ chromosome and certain regions of human chromosomes are extensively digested by either Mbo I or Alu I, despite of being regions of constitutive heterochromatin (Mezzanotte et al., 1983, 1985; Miller et al., 1983; Mezzanotte and Ferrucci, 1984). In $V$. faba, however, treatment with Mbo I or Alu I produces metaphase differentiation which is very similar to the $C$ banding pattern, i.e., neither enzyme digests $\mathrm{C}$ bands with the exception of the NOR-associated $\mathrm{C}$ band of the secondary constriction. Frediani et al. (1987) hypothetize that in $V . f a b a$ (and in general in plants indeed) $\mathrm{C}$ band regions represent an impediment to nuclease activity.

In this paper we have examined fixed metaphase chromosomes of four suitable liliaceous species with different types of C-bands, Scilla autumnalis, A. subvillosum, Muscari comosum, and $A$. cepa after digestion with some REs. In general, a good C-banding pattern is obtained in all cases; the $\mathrm{C}$ bands are resistant to all the used 
enzymes, but $\mathrm{C}$ bands localised in the nucleolar organizers can be digested by some enzymes.

\section{MATERIALS AND METHODS}

Three species of family Liliaceae are initially analysed: S. autumnalis L., A. subvillosum L., and $M$. comosum L. Miller. Bulbs of each species were collected at several natural populations in Granada (Spain) and were grown under hydroponic conditions over 2-7 days. We have also obtained commercially bulbs of $A$. cepa and analysed lateral root tips. Slides were treated follawing the technique described in Schwarzacher et al. (1980). The conventional C-banding, silver nitrate staining and fluorochrome staining were performed according to Schwarzacher et al. (1980), Kodama et al. (1980) and Schweizer $(1976,1980)$, respectively.

The restriction endonuclease-induced banding and sequential staining procedures were made as follows. In situ digestions with restriction endonucleases were carried out by adding the specific enzyme solution plus appropriate buffer to slides of mitotic preps. The concentration of endonuclease varied from 0.5 to 1 units $/ \mu l$ (table 1 ). As a control preps of the same individuals were treated with buffer alone. Both types of slides were incubated for 16 hours in a moist chamber at $37^{\circ} \mathrm{C}$. The slides were then washed with distilled water and stained with 2 per cent Giemsa in phosphate buffer at $p \mathrm{H} 6.8$ for $5 \mathrm{~min}$. After photographing good mitotic metaphases the slides were destained with Carnoy fixative for a few minutes and then sequentially stained with fluorochromes and silver nitrate as previously described. Between staining with fluorochromes and silver nitrate the preps were also destained overnight in Carnoy fixative and left a few days in absolute methanol.

\section{RESULTS}

\section{Scilla autumnalis $L$.}

S. autumnalis has a standard karyotype with $2 n=$ 14 chromosomes (fig. 1(a)). Only one NOR located in the intercalary satellite of the fifth chromosome pair exists (fig. 1(b)); and only one positive C-band is seen: again in the NOR (fig. 1(c)). This heterochromatic band is $\mathrm{CMA}_{3}$ positive (fig. 1(d)) and DAPI negative (fig. 1(e)); therefore it is assumed to be GC-rich.

After treatment of fixed metaphase chromosomes of S. autumnalis with Alu I, Rsa I and Dra I respectively the banding pattern obtained is similar in each case and similar to the C-banding pattern. That is to say, the positive C band of the NOR is resistant to these three enzymes. This is confirmed after sequential staining with Giemsa (fig. 1(f)), and fluorochromes (fig. $1(\mathrm{~g})$ ): it remains as the $\mathrm{CMA}_{3}$ positive (fig. 2(a)) and DAPI negative band. In the interphase nuclei two small chromocentres persist (fig. 2(a)), and after subsequent Ag-staining we can show that it is still possible to visualize the NOR. In constrast, after digestion with Hae III (whose target is only composed of GC pairs) and subsequent Giemsa staining no bands can be seen in the karyotype of $S$. autumnalis (fig. 1(h)), neither does any $\mathrm{CMA}_{3}$ positive band exist after sequential staining with fluorochromes (figs 1(i) and 2(b)). That is to say, the positive C band of the NOR is entirely digested by Hae III. In accordance with this observation, after Hae III digestion the interphase nuclei lack chromocentres (fig. 2(b)).

Table 1 Enzyme employed in our analyses with specification of concentration, base sequence targets and type of chromosome banding banding pattern produced by each one

\begin{tabular}{|c|c|c|c|c|c|c|}
\hline \multirow[b]{2}{*}{ Enzyme } & \multirow[b]{2}{*}{$\begin{array}{l}\text { Concentration } \\
\text { (units } / \mu \mathrm{l})\end{array}$} & \multirow[b]{2}{*}{$\begin{array}{l}\text { Recognition } \\
\text { sequence }\end{array}$} & \multicolumn{4}{|c|}{ Chromosome banding pattern } \\
\hline & & & $\begin{array}{l}\text { Scilla } \\
\text { autumnalis }\end{array}$ & $\begin{array}{l}\text { Allium } \\
\text { subvillosum }\end{array}$ & $\begin{array}{l}\text { Muscari } \\
\text { comosum }\end{array}$ & $\begin{array}{l}\text { Allium } \\
\text { cepa }\end{array}$ \\
\hline Alu I & $0 \cdot 5$ & $\mathrm{AG} / \mathrm{CT}$ & $\mathrm{C}$ & $\mathrm{C}^{*}$ & $\mathrm{C}$ & $\mathrm{C}$ \\
\hline Hae III & $1 \cdot 0$ & $\mathrm{GG} / \mathrm{CC}$ & $\mathrm{C}^{* *}$ & $\mathrm{C}^{*}$ & $\mathrm{C}$ & $\mathrm{C}$ \\
\hline Rsa I & $0 \cdot 5$ & $\mathrm{GT} / \mathrm{AC}$ & $\mathrm{C}$ & $\mathrm{C}^{*}$ & $\mathrm{C}$ & - \\
\hline Dra I & 0.75 & TTT/AAA & $\mathrm{C}$ & $\mathrm{C}$ & $\mathrm{C}$ & - \\
\hline Pvu II & 0.5 & $\mathrm{CAG} / \mathrm{CTG}$ & - & - & - & $\mathrm{C}$ \\
\hline Dde I & $0 \cdot 5$ & C/TNAG & - & - & - & $\mathrm{C}$ \\
\hline Hind III & $0 \cdot 5$ & A/AGCTT & - & - & - & $\mathrm{C}$ \\
\hline
\end{tabular}

$\mathrm{C}=\mathrm{C}$-banding pattern.

$\mathrm{C}^{*}=\mathrm{C}$-banding pattern but some of C-bands NOR-associated are digested.

$\mathrm{C}^{* *}=$ The only C-band of the species is NOR-associated and it is digested. 


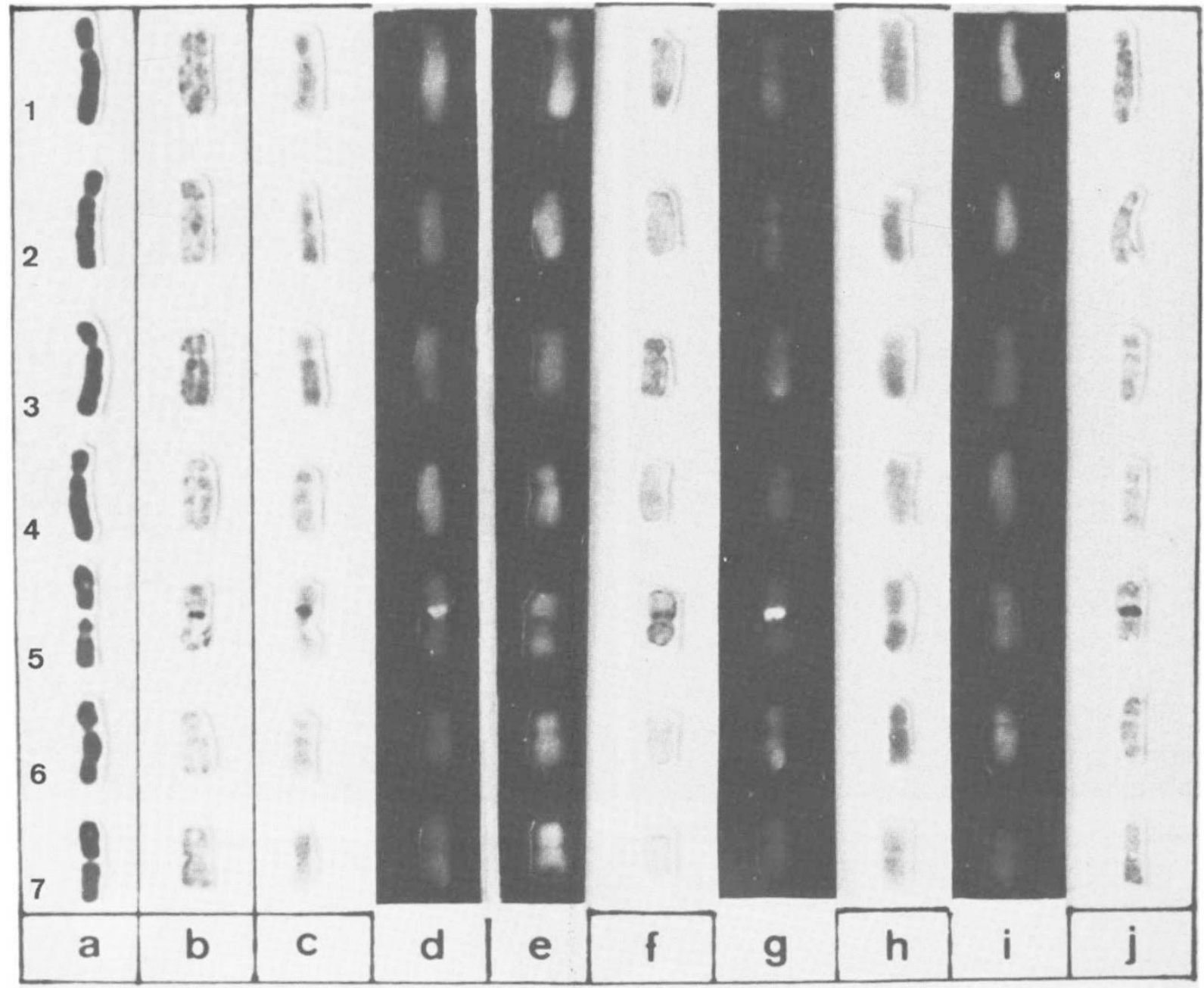

Figure 1 Haploid karyotypes of Scilla autumnalis L. showing orcein staining (a), silver staining (b), C-banding (c), CMA staining $_{3}$ (d), DAPI staining (e), Giemsa staining after Dra I digestion (f), $\mathrm{CMA}_{3}$ staining after Dra I digestion (g), Giemsa staining after Hae III digestion (h), CMA 3 staining after Hae III treatment (i) and silver staining after Hae III digestion (j). Bar represents $5 \mu \mathrm{m}$.

Finally, after sequential Ag-staining it is still possible to visualize the NOR (fig. $1(\mathrm{j})$ ). In $S$. autumnalis and all the other species analysed here the controls show that no bands can be produced with Giemsa or fluorochrome staining after incubation in buffer alone.

\section{Allium subvillosum}

A. subvillosum has a karyotype with $2 n=28$ chromosomes (fig. 3(a)). Three NORs exist in the short arm of the second, third and fourth chromosome pairs (fig. 3(c)); and in some individuals one of the chromosomes of the third pair has an inactive NOR. The chromosomes show constitutive heterochromatin regions in three positions: the centromeres, the telomeres and interstitially (fig. $3(b)$ ). The NORs show faint heterochromatic C bands on both sides of the secondary constrictions (fig. 3(b)). The fluorescence pattern of the heterochromatic regions yields information about the repetitive DNA located in them: it is GC-rich because all the chromosomes revealed $\mathrm{CMA}_{3}$ positive and DAPI negative bands in the same positions as the $\mathrm{C}$ bands. This is particularly clear in the case of the four big $\mathrm{CMA}_{3}$ positive and DAPI 

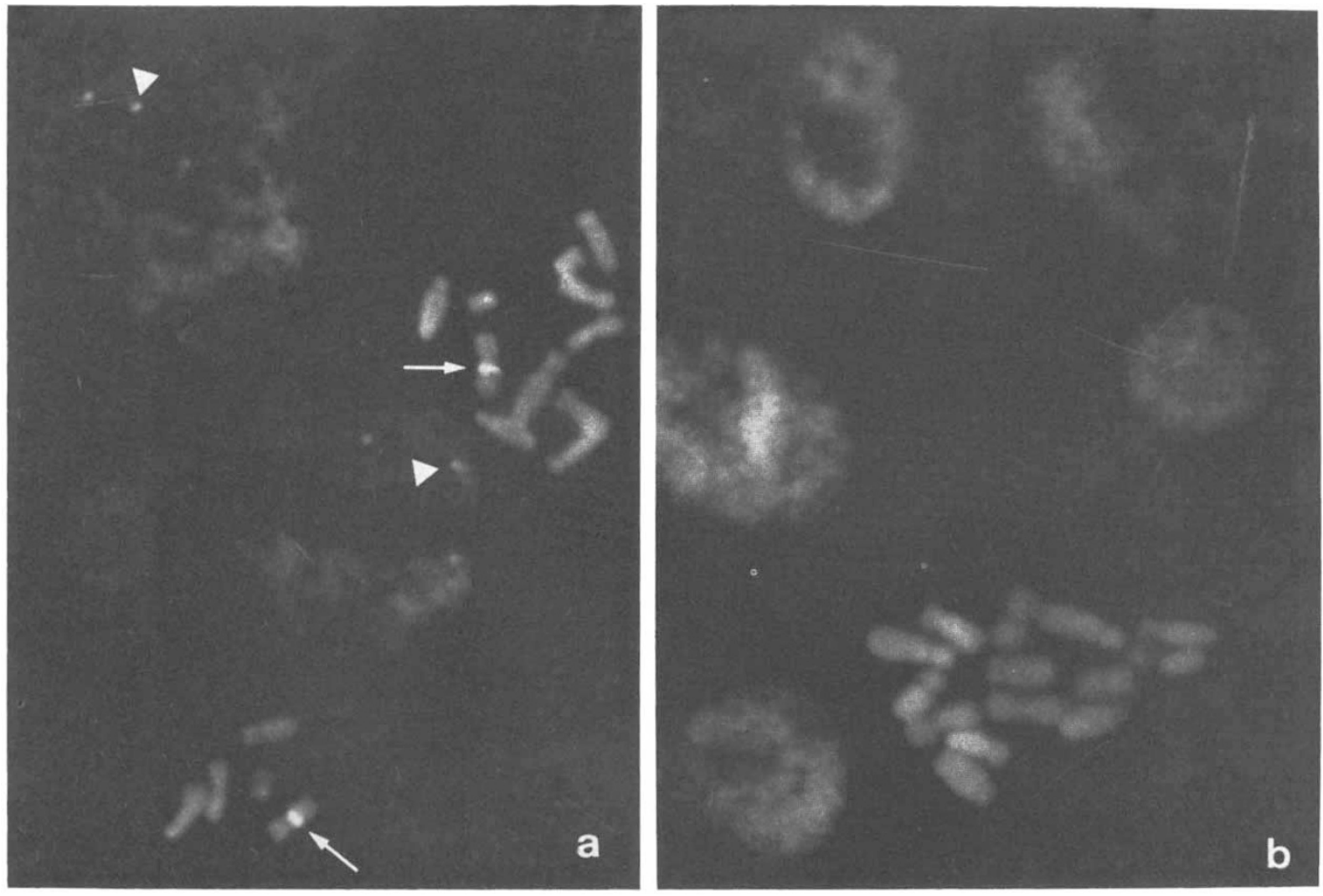

Figure 2 Chromosomes and interphase nuclei of Scilla autumnalis treated with the restriction endonucleases Alu I (a), and Hae III (b) followed by $\mathrm{CMA}_{3}$ staining. Arrowheads in (a) indicate the $\mathrm{CMA}_{3}$ positive chromocentres in interphase nuclei and arrows point to the $\mathrm{CMA}_{3}$ positive band in the fifth chromosome pair.

negative blocks located in chromosomes 1, 5, 9 and 11 (figs 3(d) and 3(e)). Three smaller double $\mathrm{CMA}_{3}$ positive blocks are also present in the NORs (fig. 3(d)).

The heterochromatic bands of $A$. subvillosum not localized in the NORs are resistant to digestion with all four endonucleases used here (Hae III, Alu I, Rsa I and Dra I) (figs 3 and 4). In contrast, the $\mathrm{C}$ bands localised in the NORs behave in a different way. After Dra I digestion all the heterochromatic bands of the three NORs (even of the inactive NOR) are visible with $\mathrm{CMA}_{3}$ (figs $3(\mathrm{~m})$ and $4(\mathrm{~b}))$, and in the case of the active NORs it is possible to sequentially visualize the NORs with Ag-staining (fig. 4(c)). However, after digestion with enzymes whose targets only have GC pairs (Hae III) or are GC-rich (Alu I and Rsa I) the heterochromatic bands of certain NORs are digested: Hae III digests the $\mathrm{C}$ bands of the NOR on the second chromosome pair (figs $3(\mathrm{~h}$ ) and 3(i)), Alu I digests the C-bands of the NOR on the third pair (figs $3(\mathrm{f})$ and $3(\mathrm{~g})$ ) and Rsa I digests the bands in the fourth pair (figs $3(\mathrm{j}), 3(\mathrm{k}), 4(\mathrm{~d})$ and $4(\mathrm{e}))$. Finally, as in the case of $S$. autumnalis, it is possible to sequentially visualize the NORs with Ag-staining (fig. 4(f)), even after the associated C bands have been digested.

\section{Muscari comosum $L$.}

$M$. comosum has a standard karyotype with $2 n=18$ chromosomes (fig. 5(a)). The long arm of the first chromosome pair shows four or five interstitial heterochromatic bands (fig. 5(b)). These heterochromatic areas are seen as two big chromocentres in the interphase nuclei. After Agstaining it is possible to visualize a faint NOR in a small pair of metacentric chromosomes (figure not shown). The $\mathrm{C}$ bands of the first pair are DAPI positive (fig. 5(c)): this suggests that the big heterochromatic bands of $M$. comosum are ATrich.

After treatment of fixed metaphase chromosomes of $M$. comosum with Hae III, Alu I and 


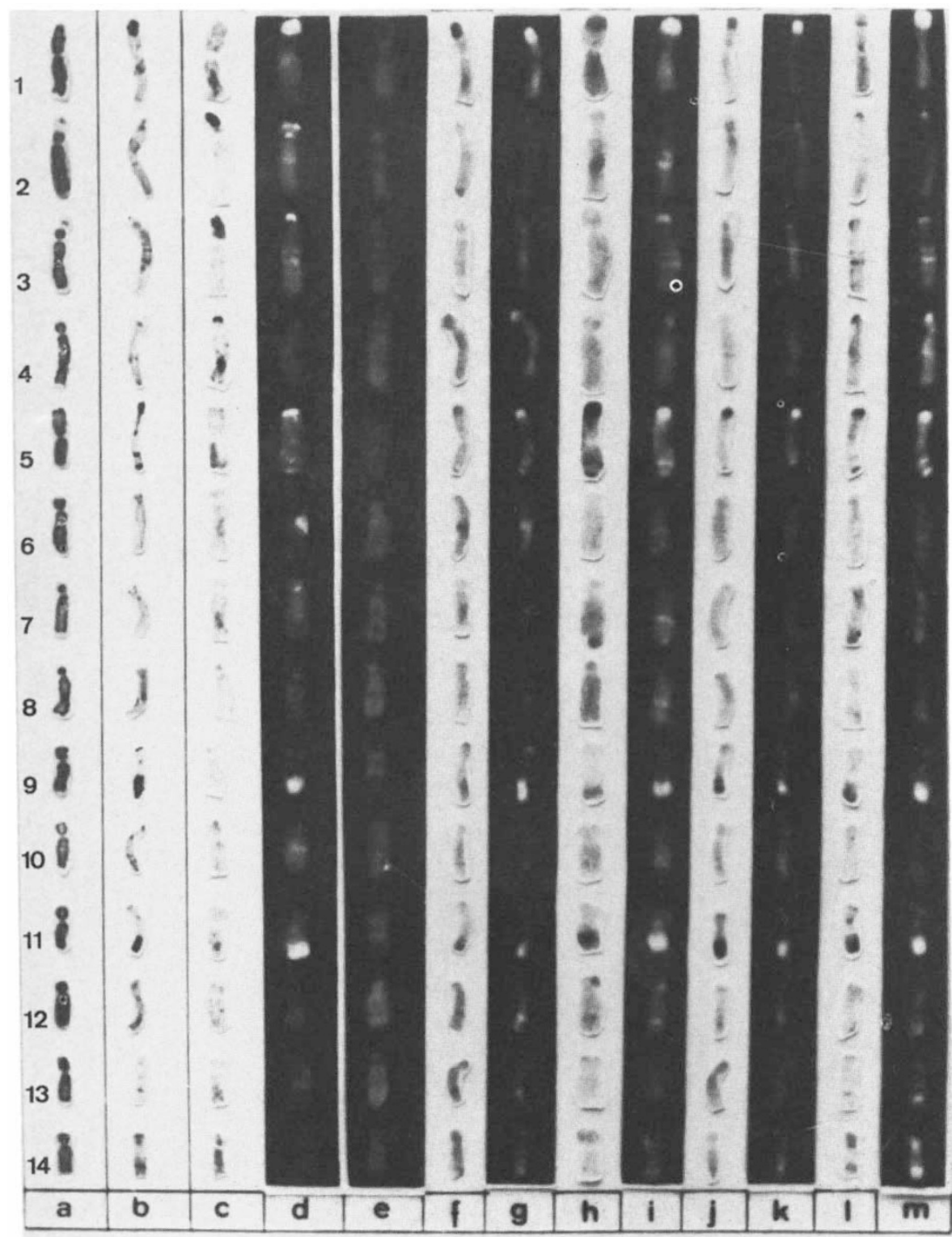

Figure 3 Haploid karyotypes of Allium subvillosum L. showing orcein staining (a), C-banding (b), silver staining (c), sequential $\mathrm{CMA}_{3}$ and DAPI staining (d)-(e) and sequential Giemsa and CMA staining after treatment with Alu I (f)-(g), Hae III (h)-(i), Rsa I (j)-(k) and Dra I (1)-(m). Bar represents $15 \mu \mathrm{m}$.

Rsa I, and also after treatment with Dra I the heterochromatic bands of the first pair are resistant. So, it is possible to visualize these bands with the four enzymes after staining with Giemsa (fig. 5(d)) and DAPI (fig. 5(e)). Furthermore, in all cases the chromocentres are clearly visible (fig. $5(\mathrm{f})$ ). Because the C-band associated with the NOR of $M$. comosum is very small it was not possible to carry out a detailed analysis of its digestion pattern as in the case of $S$. autumnalis and $A$. subvillosum.

\section{Allium cepa}

A. cepa has $2 n=16$ chromosomes; C bands are localised interstitially, and at the centromeres and 

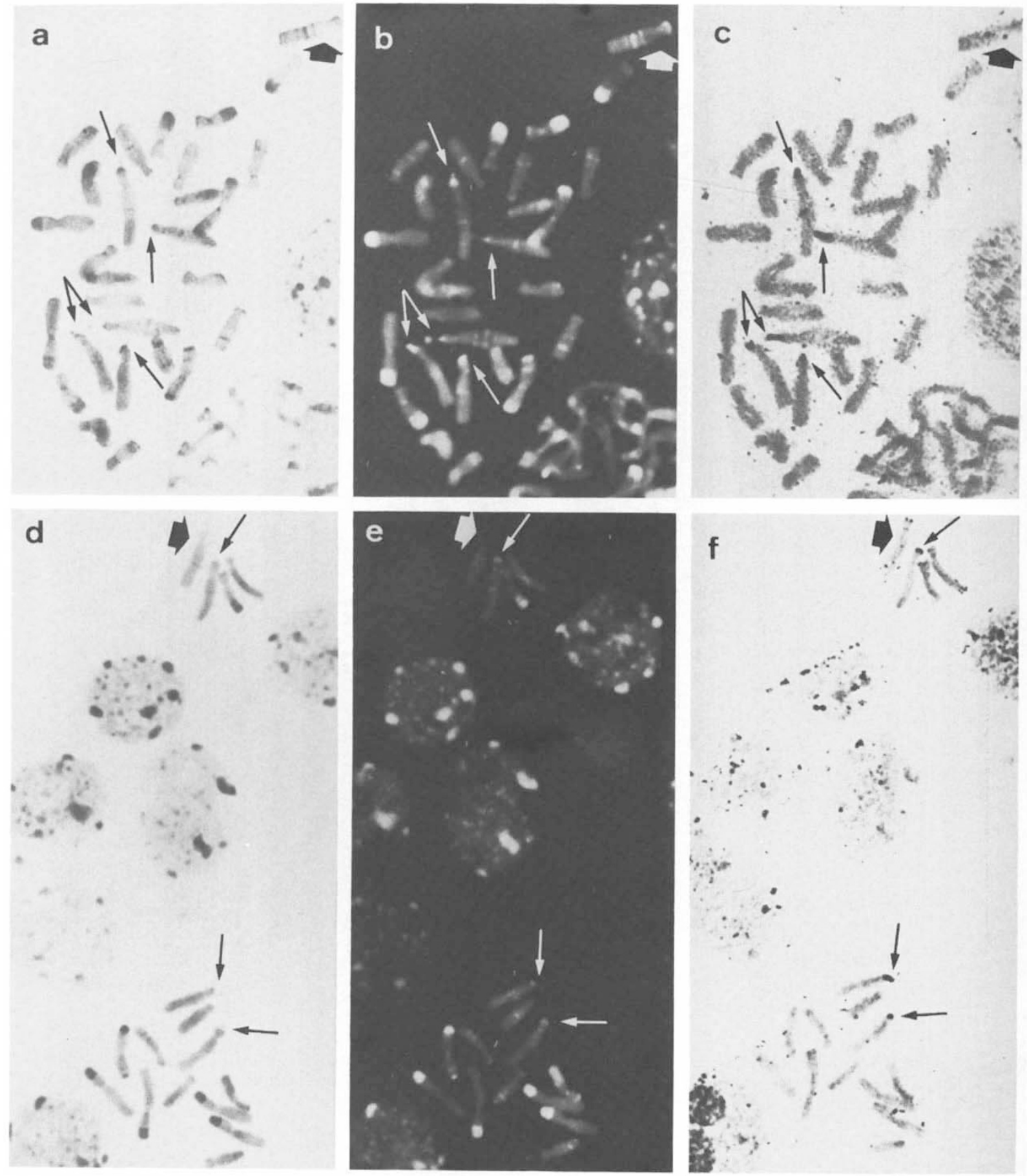

Figure 4 Metaphase chromosomes of Allium subvillosum treated with Dra I (a)-(c) followed by sequential Giemsa (a), CMA and silver (c) staining. Arrowheads point to one chromosome with an inactive NOR and arrows indicate the active NOR chromosomes. Chromosomes of the same species digested with Rsa I (d)-(f, incomplete cell) followed by sequential staining with Giemsa (d), $\mathrm{CMA}_{3}$ (e) and silver nitrate (f). See the chromocentres in the interphase nuclei. Arrows indicate the NORs bearing chromosomes in which the C-bands NOR-associated are resistant to digestion; arrowheads point to one NOR chromosome in which the C-bands are digested (see (d)-(e)) but the NOR visualization persists (f). 

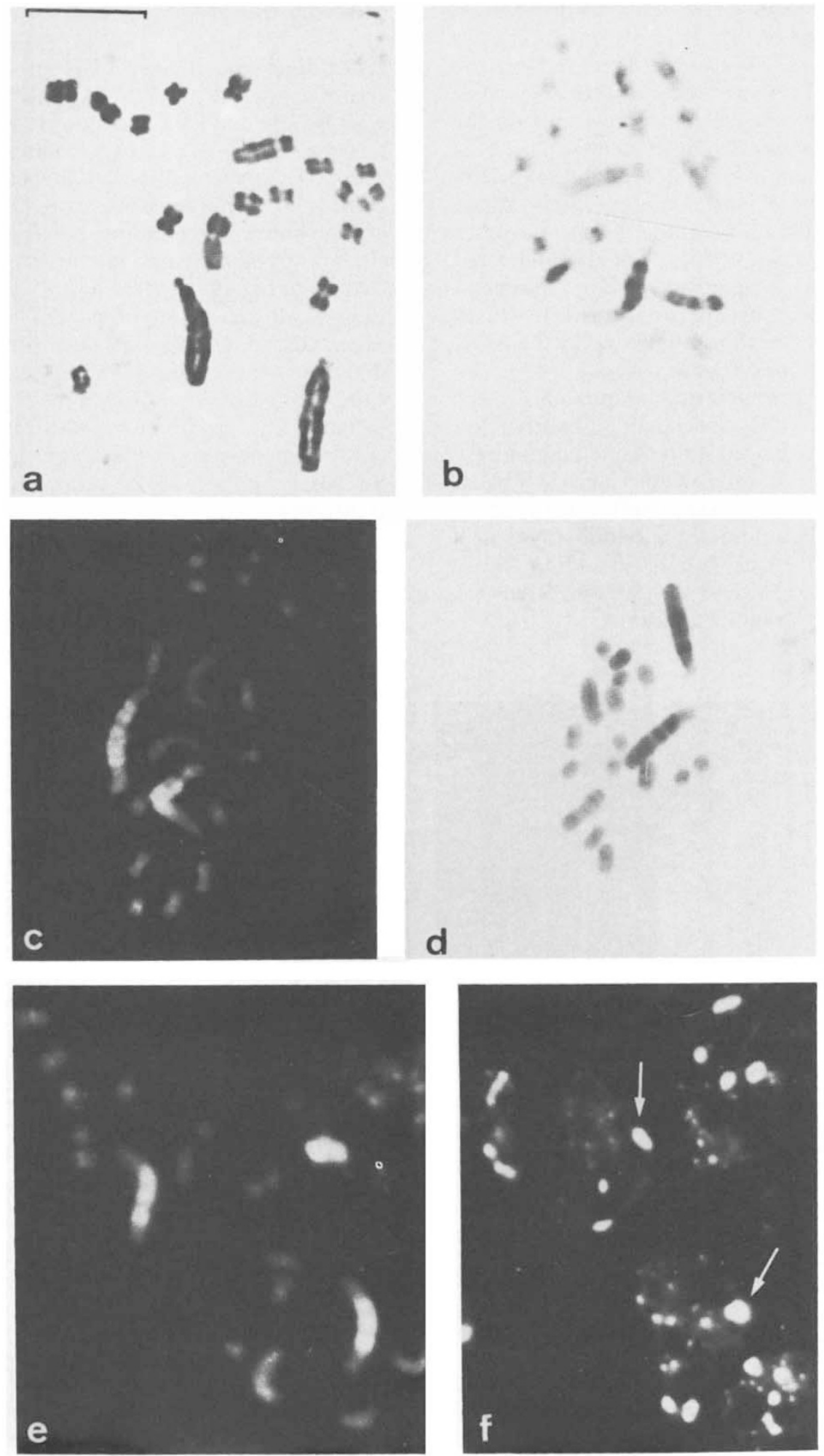

Figure 5 Mitotic chromosomes of Muscari comosum after orcein staining (a), C-banding (b) and DAPI staining (c), and after Dra I treatment followed by staining with Giemsa (d) and DAPI (e)-(f). Note the big positive DAPI chromocentres resistant to enzyme digestion (f). Bar represents $5 \mu \mathrm{m}$. 
telomeres (Vosa, 1976; Stack et al., 1979). A variable number of NORs (1-4) exists in this species (Sato, 1981). These NORs are localised on the short arm of a pair of subtelocentric chromosomes (the regular nucleolar chromosomes) and on the short arm of the smallest submetacentric pair. This variability is thought to be a consequence of NORs "jumping" between terminal heterochromatic blocks of the above mentioned chromosomes (Schubert and Wobus, 1985). Tandemly repeated satellite DNA is located in all the telomeric chromosome regions except those associated with the secondary constrictions, that is to say the NOR bearing chromosomes (Barnes et al., 1985). We have observed that telomeric $\mathrm{C}$ bands of $A$. cepa (including the NOR-associated C-bands) are $\mathrm{CMA}_{3}$ positive. After digestion of fixed metaphase chromosomes of $A$. cepa with Hae III, Pvu II, Hind III, Dde I, Alu I and Dra I and staining with $\mathrm{CMA}_{3}$ all the telomeric $\mathrm{C}$ bands are clearly visible (figs 6(a) and 6(b)), including those associated with the NORs (we have only analysed bulbs with the two regular NORs).

\section{DISCUSSION}

After digestion of fixed liliaceous plant chromosomes is possible to obtain a good banding pattern with REs which have recognition sequences of six bases pairs (bp) (e.g., Dra I, Hind III) as well as those with recognition sequences of four bp (Hae III, Alu I, Rsa I) or five bp (Dde I). In animal chromosome the banding patterns produced by six bp-target enzymes are in general very poor (Miller et al., 1983; Mezzanotte et al., 1983, 1985; Bianchi et al., 1985). In general the C-bands of liliaceous plant are not susceptible to enzyme digestion. In vertebrates and insects some C-bands can be digested significantly with some REs. For instance, the proximal heterochromatin of the $\mathrm{X}$ chromsome of Drosophila melanogaster, is digested by Alu I and Hae III (Mezzanotte, 1986); the centromeric regions of mouse chromosomes are digested by BstN I (Miller et al., 1984), and the paracentromeric heterochromatic areas of human chromosome 1, 9 and 16 can be digested using Hinf I (Bianchi et al., 1985). In accordance with
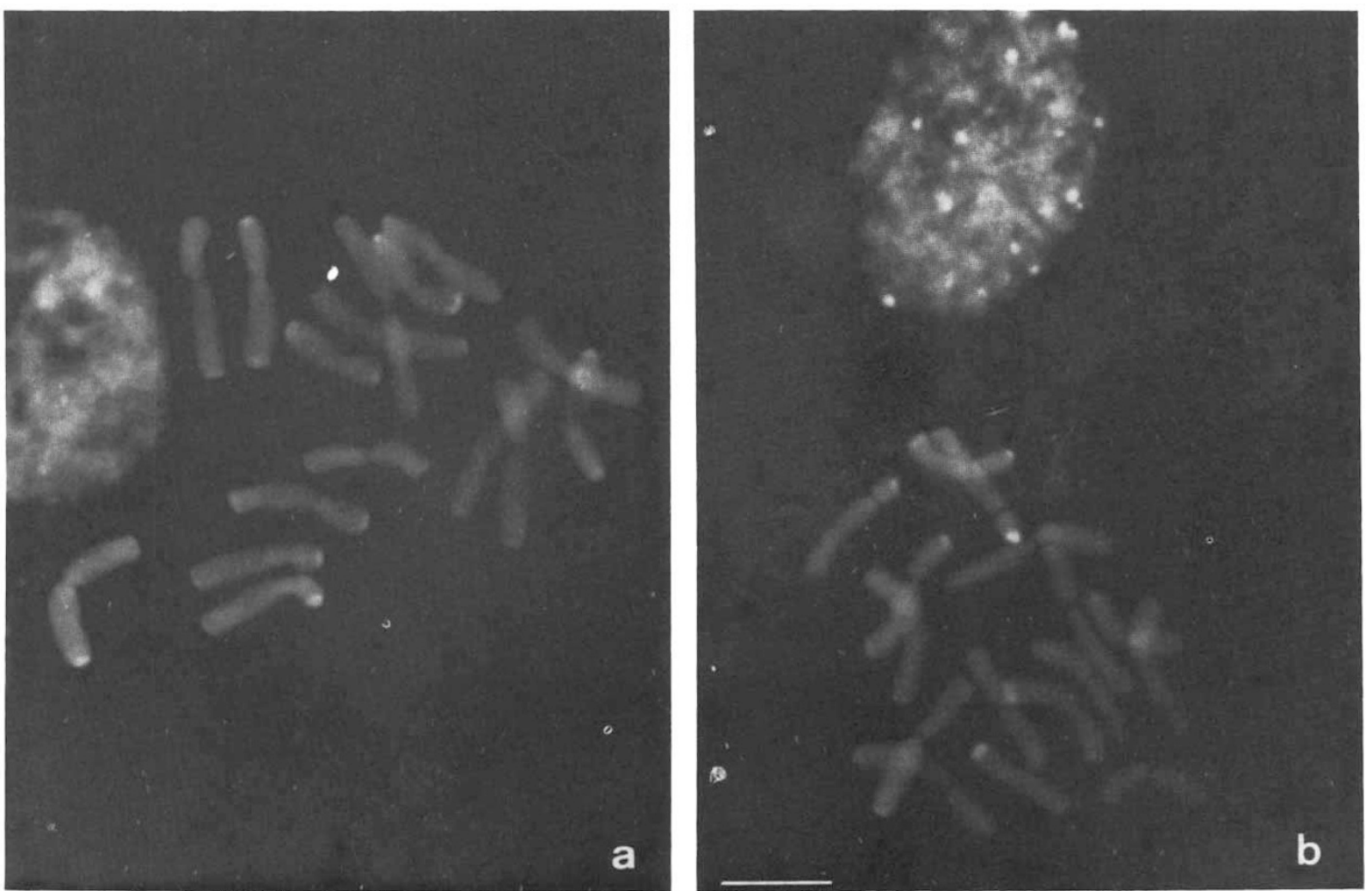

Figure 6 Allium cepa mitotic chromosomes after digestion with Hind III (a) and Hae III (b) followed by CMA 3 staining. Bar represents $10 \mu \mathrm{m}$. 
these observations, Alu I and Hae III have numerous restriction sites in the DNA located in the proximal heterochromatin of the $\mathrm{X}$ chromosome of D. melanogaster, BstN I has numerous targets in the mouse centromeric satellite DNA, and Hinf $I$ in the human satellite DNA III located in the above mentioned chromosomes (data obtained from the Gen Bank). Thus it can be surmised that certain regions of animal heterochromatin have recognition sequences for specific REs and are susceptible to attack by these REs in fixed chromosomes. In liliaceous species we have analysed the possibility that the resistence of the C-bands to endonuclease attack could be the absence of specific base sequence targets in these banding regions. However, this is unlikely in $A$. subvillosum and $M$. comosum, and cannot be the case in A. cepa whose heterochromatin is well characterised and known to contain target sequences for some enzymes used like Hae III, Pvu II, Alu I and Hind III (Barnes et al., 1985 and EMBL Nucieotide Sequences Data Library). It could be argued that the REs do not appear to affect the heterochromatic of $A$. cepa only because the fragments generated after their action are very large. However in a theoretical comparative analysis between the satellite DNA of $A$. cepa and those of the above mentioned animal species it is found that in both cases fragments of similar size would be generated after digestion with specific enzymes (table 2). Furthermore these fragments are all under the critical maximum of $350 \mathrm{bp}$ thought to be necessary for in situ DNA extraction (Miller et

Table 2 Hypothetical size of the fragments after digestion of some animal and plant DNA satellites with some restriction endonucleases

\begin{tabular}{lll}
\hline Locus & $\begin{array}{l}\text { Restriction } \\
\text { enyme }\end{array}$ & $\begin{array}{l}\text { Size of fragments } \\
\text { after digestion (base pairs) }\end{array}$ \\
\hline HUMPPD17 & Hinf I & $10,12,13,16,18,18,26,39$ \\
MUSRSSAT & BstN I & $5,187,234$ \\
DROSAT359 & Alu I & 173,186 \\
& Hae III & 359 \\
ACSAT1 & Alu I & 15,365 \\
& Hae II & 175,205 \\
& Dde I & no fragments \\
& Dra I & no fragments
\end{tabular}

HUMPPD17 $=$ Human satellite III related DNA (clone ppd 17).

MUSRSSAT $=$ Mouse satellite repeat unit.

DROSAT359 $=$ Drosophila $1.688 \mathrm{~g} / \mathrm{ml}$ satellite repeating unit. ACSAT1 = Allium cepa satellite DNA repeat (clone 8062). HUMPPD17, MUSRSSAT and DROSAT359 information have been obtained from the GenBank and ACSAT1 information have been obtained from the EMBL Nucleotide Sequence Data Library. al., 1983 and Mezzanotte, 1986). The resistance of the heterochromatin of these liliaceous plants to REs can be explained by any of the following hypotheses: (i) plant heterochromatin has a higher degree of methylation than animal heterochromatin and this can mask the recognition sequences of some REs; (ii) the optimal size of the fragments extracted from plant chromosomes is smaller than those in animal chromosomes, and (iii) plant heterochromatin in general, unlike animal heterochromatin, is not accessible to REs due to its different structure and/or organization. Considering the first hypothesis, it is known that some satellite DNAs, such as that of the mouse or $S$. sibirica contain more methylated cytosine residues than main-band DNA. In the case of the mouse, the satellite DNA contains almost twice as much methylated cytosine as the rest of the DNA, and in Scilla the number of methylated C residues in the satellite DNA far exceeds that of any other eukaryotes (Deumling, 1981). Although the degree of methylation of the telomeric DNA of $A$. cepa is not known, it has been shown that DNA isolated from these satellite regions can be cut extensively with for instance Alu I and Hae II (Barnes et al., 1985). Thus the in situ resistance of this chromatin to REs cannot definitively be attributed to methylation. In relation, to the second hypothesis, we are now doing codigestion experiments with two or more REs in order to obtain smaller fragments and to determine whether these can be extracted from plant heterochromatin in situ. So far the results of such experiments in $A$. cepa suggest that heterochromatin remains resistant after enzyme codigestion (unpublished data). Our observation of resistance of plant heterochromatin to digestion with an enzyme for which it has sufficient targets would then support the conclusion that plant and animal heterochromatin may well be different. Many differences between plant and animal chromatin have been described, e.g., plant chromosomes are more highly condensed than animal chromosomes and contain a higher propertion of repetitive DNA (Greilhuber 1977; Nagl, 1976). Plant chromatin lacks the DNAase hypersensitive sites present in animal chromatin (Murray and Kennard, 1984) and the high mobility group (HMG) proteins vary markedly between kingdoms (Spiker, 1985). In addition there may be some differences in the organisation of the chromatin fibres between plants and animals (Schweizer 1979). On the other hand, Burkholder and Weaver (1975) have found that DNAas I digests extended chromatin faster than condensed chromatin possibly because of the higher DNA-protein complexes in hetero- 
chromatin. It is also known that the heterochromatin can differ in relation to euchromatin not only in DNA sequences but also in the presence of certain proteins which preferentially bind to supercoiled satellite DNA (Commings et al., 1973; Hsich and Brutlag, 1979).

Our finding that the NOR-associated $\mathrm{C}$ bands of S. autumnalis and A. subvillosum can be digested by specific REs is indicative of two facts. Firstly, the structure of plant heterochromatin per se is not necessarily an absolute and insurmountable impediment to RE action. Secondly, there are at least two types of heterochromatin in plants: that which is not accessible to RE action (all the general C-bands) and that which is accessible (the C-bands associated to the NORs). But why should the NOR-associated C-bands exhibit a different digestion pattern to the other C-bands? It is possible that the former are susceptible to nuclease attack because their structure and/or organisation is dissimilar to the C-bands of the rest of the karyotype. Alternatively the NOR-associated C-bands could be RE-sensitive by virtue of their location on the chromosome, i.e., such that these bands are more accessible than the others to REs. In relation with the first explanation, it is known that certain nonhistone proteins are specifically associated with the NOR chromatin (Schwarzacher et al., 1978; Likovsky and Smetana, 1981; Spector et al., 1984; Schmid, 1982; Hubbel, 1985). These proteins can alter the structure/organisation of an NOR (including the neighbouring heterochromatin) such that it becomes susceptible to the action of specific REs. Considering the second explanation the NOR-associated C-bands would be more accessible to REs because they are localised close to a region which is relaxed and active throughout much of the cell cycle. Recently Sato (1988) has also shown, using acridine orange staining after C-banding, that the NOR-associated heterochromatic segments of some plants were differentiated from other heterochromatic segments of the chromosomes. On the other hand, the observation that NORs can be visualised with $\mathrm{Ag}$ even after digestion of the associated heterochromatin can be interpreted as follows. Firstly it is possible that $\mathrm{Ag}$ and Giemsa do not stain identical chromosome regions. Thus the digestion of C-bands close to the NOR would not affect the rDNA with NOR activitv. There is some cytological evidence that the C-bands are adjacent to and distinct from the active NOR (Pathak, 1980; Schubert, 1984), although in the majority of our observations C. banding appears to overlap with $\mathrm{Ag}$ banding. A second explanation could be that the REs extract some of the DNA of the NOR without disrupting the silver-sensitive protein, such that the latter persist and allow subsequent staining and visualisation of the NOR.

The restriction endonuclease banding technique we have applied in this work can be very useful in the cytogenetic analysis of plants because although it does not allow G-banding it does permit the sequential analysis and characterisation of chromosomes by C-banding (or similar) and fluorochromes (thus we can obtain information about heterochromatin and its composition). Finally, this technique also yields data concerning the NORs of the species studied. Conversely the application of conventional C-banding to fixed chromosomes of plants only allows the subsequent staining with fluorochromes and not with silver nitrate. This is possibly due to the fact that Cbanding extracts more DNA and proteins and acts less specifically (Holmquist, 1979; Hancock and Sumner, 1982; Burkholder and Duczek, 1982) than restriction enzyme banding, thereby totally eliminating rDNA and its associated proteins and altering the structure of the NOR. One final comment about this technique: if only one staining is performed after REs digestion, it is better to use fluorochromes rather than Giemsa. After Giemsa staining some C-bands are not visualized but after fluorochrome staining it is clear that these bands persist (see figs $3(\mathrm{f})-(\mathrm{m})$ and $4(\mathrm{a}),(\mathrm{b}),(\mathrm{d}),(\mathrm{e})$ ). In order to prevent making mistakes about the effects of REs on fixed chromosomes it is therefore necessary to visualize the chromosomes with fluorochrome staining.

Acknowledgements This work was supported by a grant of Comisión Interministerial de Ciencia y Tecnología No. PB87. 0881 . We thank Dr J. L. Oliver for his contribution in the aspects related with the base sequence data analyses and for his valuable comments. We also thank Helen Vaughan who improved the English presentation of the manuscript.

\section{REFERENCES}

BARNES, S. R., JAMES, A. M. AND JAMiESON, G. 1985. The organisation, nucleotide sequence, and chromosomal distribution of a satellite DNA from Allium cepa. Chromosoma, 92, 185-192.

BIANCHI, M. S., BIANCHI, N. O., PANTELIAS, G. E. AND WOLFFS, G. 1985. The mechanism and pattern of banding induced by restriction endonucleases in human chromosomes. Chromosoma, 91, 131-136.

BURKHOLDER, G. D. AND DUCZEK, L. L. 1982. The effect of the chromosome banding techniques on the histone and nonhistone proteins of isolated chromatin. Can. J. Biochem., 60, 328-337. 